\title{
Endovascular Treatment of Iliac Vein Compression (May-Thurner) Syndrome: Angioplasty and Stenting with or without Manual Aspiration Thrombectomy and Catheter-Directed Thrombolysis
}

\author{
Halil Bozkaya, MD, ${ }^{1}$ Celal Cinar, MD, ${ }^{1}$ Serkan Ertugay, MD, ${ }^{2}$ Mehmet Korkmaz, MD, ${ }^{3}$ \\ Serkan Guneyli, MD, ${ }^{4}$ Hakan Posacioglu, MD, ${ }^{2}$ and Mustafa Parıldar, MD ${ }^{5}$
}

\begin{abstract}
Purpose: May-Thurner syndrome (MTS) is a rare clinical entity featuring venous obstruction of the left lower extremity. The aim of the present study was to report our experience with MTS and to evaluate the utility of treatment using endovascular techniques.

Materials and Methods: We retrospectively analyzed data on 23 MTS patients (21 females, two males; mean age $44 \pm$ 15 years). Eighteen patients presented with deep vein thrombosis (DVT) and five with symptoms associated with chronic venous hypertension (CVH). DVT patients were treated via thromboaspiration, catheter-directed thrombolysis, and angioplasty; followed by stent placement. CVH patients were treated with angioplasty and stent placement alone. All patients were followed-up using Doppler ultrasonography and computed tomography venography.

Results: Complete left common iliac vein patency was achieved in 21 of the 23 patients (technical success rate: 91,3\%). Complete thrombolysis was attained by 14 of the 18 DVT patients (77.7\%). The mean clinical and radiological follow-up time was $15.2 \pm 16.1$ months. Upon follow-up, complete symptomatic regression was observed in 19 of the 23 patients (82.6\%). Stent patency was complete in 19 of the 21 patients (90.4\%) who received stents. Restenosis occurred in two
\end{abstract}

${ }^{1}$ Division of Interventional Radiology, Department of Radiology, Ege University, School of Medicine, Izmir, Turkey ${ }^{2}$ Department of Cardiovascular Surgery, Ege University, School of Medicine, Izmir, Turkey

${ }^{3}$ Department of Radiology, Dumlupinar University, Faculty of Medicine, Kutahya, Turkey

${ }^{4}$ Department of Radiology, Bulent Ecevit University, Faculty of Medicine Zonguldak, Turkey

${ }^{5}$ Division of Interventional Radiology, Department of Radiology, Ege University, School of Medicine, Izmir, Turkey

Received: September 22, 2014; Accepted: December 18, 2014 Corresponding author: Halil Bozkaya, MD. Division of Interventional Radiology, Department of Radiology, Ege University, School of Medicine, 35100 Bornova, Izmir, Turkey Tel: +90-232-3903275, Fax: +90-232-3420001

E-mail: halilbozkaya@yahoo.com patients. No treatment-related mortality or morbidity was observed.

Conclusion: Endovascular treatment of MTS is safe and effective and reduces symptoms in most patients, associated with high medium-term patency rates.

Keywords: May-Thurner syndrome, deep vein thrombosis, stent, thrombolysis.

\section{Introduction}

May-Thurner syndrome (MTS) is a rare clinical entity featuring venous obstruction of the left lower extremity. MTS is characterized by chronic pulsatile compression of the left iliac vein in the region between the right common iliac artery and the lumbar vertebral body. Such compression triggers development of a form of collagen scar that, over time, causes complete or partial occlusion of the iliac vein. The anatomic variation associated with MTS increases the incidence of left-sided deep venous thrombosis (DVT), which is 3-8-fold more frequent on the left side than the right. ${ }^{1-4)}$ Anticoagulation therapy alone is ineffective in MTS patients. More aggressive treatment is required to prevent rethrombosis and to eliminate the long-term complications of post-thrombotic syndromee. . $^{5,6}$

Traditional surgical treatments for MTS are outdated. Endovascular treatment of DVT caused by MTS is both

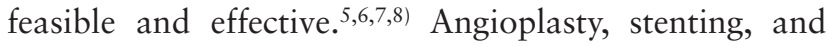
selective thrombolysis are now the standard treatments for symptomatic MTS.

The purpose of the present study was to report our experience with MTS and the treatment efficacies afforded by endovascular techniques including catheterdirected thrombolysis (CDT), manual aspiration thrombectomy (AT), stent placement, and angioplasty. 


\section{Materials and Methods}

We retrospectively analyzed data collected between April 2009 and January 2014 on 23 MTS patients treated using endovascular techniques. We reviewed patient demographics, symptoms, procedural features, and radiological and clinical outcomes. The procedural aspects were the number and size of stents used, whether thrombolysis was employed, and whether treatment featured concurrent vena cava filter placement. Written informed consent was obtained from each patient after the purpose and the risks of treatment were fully explained.

All patients exhibited radiographic evidence of iliac vein stenosis caused by MTS upon computed tomography $(\mathrm{CT})$ venography or a combination of duplex ultrasound and venography. Radiographic criteria for MTS diagnosis were $>50 \%$ luminal stenosis of the vein, and loss of contrast density at the point at which the iliac artery crossed the vein (confirmed via venography). DVT was diagnosed using color Doppler ultrasonography (CDU) and confirmed via venography performed during endovascular treatment of all patients. Technical success was defined as successful restoration of antegrade flow, with $<30 \%$ residual stenosis or thrombus. Iliac vein patency was defined as uninterrupted flow apparent on CDU or venography. Clinical success was defined as improvement in and resolution of symptoms on follow-up visits.

Of our patients, $21(91,3 \%)$ were female and 2 male. Mean patient age was $44 \pm 15$ years (range, $18-68$ years). All patients were symptomatic. Sixteen patients presented with acute DVT (8 iliofemoral; eight iliofemoropopliteal); two with subacute DVT (one iliofemoral, one iliofemoropopliteal) chracterized by swelling of and pain in the left lower extremity; four with symptoms of chronic venous hypertension including varicosities and lower limb swelling in the pelvic, inguinal, or thigh region; and one with persistent rectal bleeding caused by rectal mucosal venous congestion. Six patients exhibited risk factors for DVT development. These were a previous history of DVT $(\mathrm{n}=1)$; postoperative status $(\mathrm{n}=2)$; postpartum status $(\mathrm{n}=1)$; oral contraceptive $(\mathrm{n}=1)$ and the Factor V Leiden mutation $(\mathrm{n}=1)$. No patient had a symptomatically indicated pulmonary embolism.

Vascular access was established via ultrasonographyguided puncture of the ipsilateral popliteal vein $(n=18)$ or femoral vein $(\mathrm{n}=5)$. A vascular sheath was introduced into each vein and ascending venographic data obtained. Patients with acute and subacute DVT were initially treated via CDT and manual AT using 7-F guiding catheters (Mac1; Boston Scientific, Natick, Massachusetts, USA). CDT featured either a multi-sided hole catheter (5F Aortic Flush; Boston Scientific, USA) or ultrasound-accelerated
CDT using the EKOS catheter (EKOS Corporation, Bothell, Washington, USA). We used multisided hole catheters to treat seven patients and EKOS catheters to treat a further eleven. Infusion catheters were passed through thrombotic occlusions of the left common iliac vein. Recombinant human tissue plasminogen activator ( $\mathrm{tPA}$ ) alteplase (Actilyse; Boehringer Ingelheim GmbH \& Co., Ingelheim, Germany) was administered as an initial 5-mg bolus followed by continuous infusion at $1 \mathrm{mg} / \mathrm{h}$ during each procedure. In addition, heparin was infused via the hemostatic sheath to maintain the aPTT in the range 60-90 s. Inferior vena cava (IVC) filter placement was employed in four patients considered to be at very high risk of pulmonary embolism (PE). These patients were our first MTS cases treated via endovascular methods. Vena cava filters were not placed in later cases because of the low risk of PE associated with MTS.

Venography was repeated after $24 \mathrm{~h}$ and CDT therapy was continued for a further $24 \mathrm{~h}$ if clot lysis was inadequate. Such therapy was discontinued if any contraindicative complication developed.

Patients with venographically determined iliac vein stenosis or occlusions were treated via angioplasty and stenting. Stent size was determined by measuring the diameter of the patent distal common iliac vein and stent length was chosen to ensure complete lesion coverage. Each stent was deployed 1-2 cm into the inferior vena cava if the lesion was located less than $2 \mathrm{~cm}$ from the orifice of the left common iliac vein. Self-expanding nitinol stents (Epic; Boston Scientific, USA; and Smart; Cordis, Miami, Florida, USA); stainless steel stents (Wallstent; Boston Scientific, USA); and XL stents (Sinus XL; Optimed, Ettlingen, Germany) were employed. Balloon dilatation was performed routinely after iliac vein stenting. The diameter of the balloon catheter was $12 \pm 2 \mathrm{~mm}$ (Powerflex; Cordis, USA).

All patients received 10,000 units of heparin at the start of each procedure, and systemic heparinization was continued after the procedure to maintain the aPTT at 60-90 s for 3 days. All patients were prescribed oral warfarin sodium for 6 months to maintain the international normalized ratio in the range 2.0-3.0. All patients were instructed to wear graduated compression stockings with which they were provided.

Follow-up clinical and imaging evaluations were initially performed at 1 and 6 months, and yearly thereafter. Follow-up imaging featured primarily CDU. A few patients were re-imaged using CT or repeat venography because it was difficult to obtain adequate images of the stents using CDU alone.

Data are expressed as means \pm standard deviations for continuous variables and as percentages for categorical variables. 

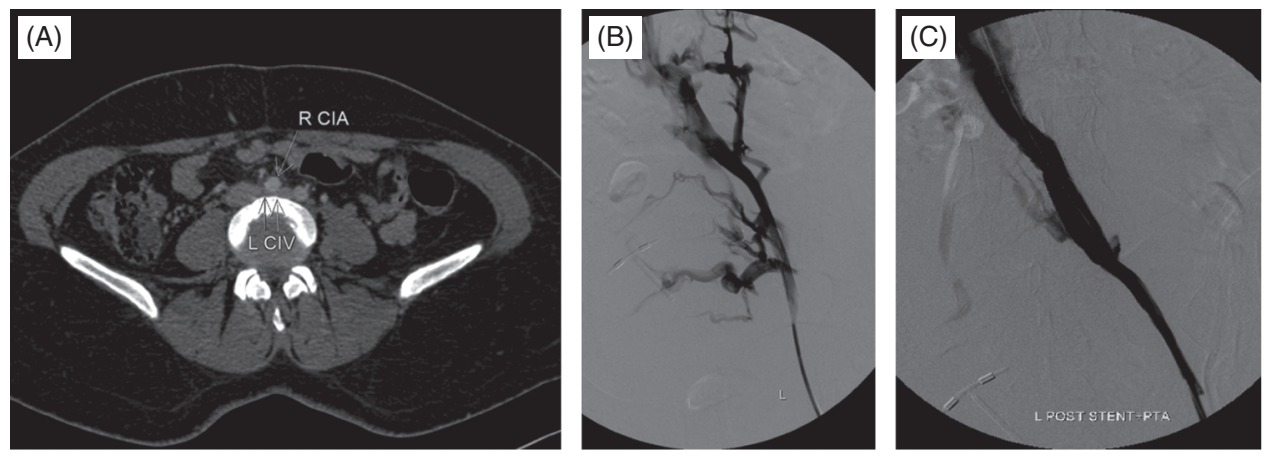

Fig. 1 A 44-year-old female who presented with the left lower extremity and inguinal varicosities. (A) A transverse computed tomography image shows the right common iliac artery (single arrow) compressing the left common iliac vein (double arrow). (B) Initial venography revealed significant stenosis of the left common iliac vein with extensive cross-pelvic and hypogastric collateralization. (C) Venography performed after stent implantation and angioplasty revealed complete resolution of collateral filling with good flow through the femoral and iliac system into the IVC.

\section{Results}

Venography performed during endovascular treatment of MTS revealed significant stenosis of the left common iliac vein in 15 of $23(65.2 \%)$ patients. In 8 of the $23(34.8 \%)$ patients, total occlusion of the left common iliac vein (LCIV) was evident. All patients presenting with venous varices exhibited common iliac vein stenosis. Restoration of complete LCIV patency was successful in 21 of the 23 patients (technical success rate: 91.3\%). In two DVT patients, the occluded iliac vein could not be reached even with the aid of a guide wire; no stents were placed.

Thrombolysis was performed for $24-48 \mathrm{~h}$ (mean, $30.5 \pm$ $16.6 \mathrm{~h}$ ) in 18 patients with acute and subacute DVT. The average tPA dose was $31.25 \pm 16.1 \mathrm{mg}$ (range, $20-50 \mathrm{mg}$ ). Thrombus removal was complete in 14 patients $(77.7 \%)$, Four of the eighteen patients had residual clots upon cessation of thrombolysis (partial thrombolysis; $22.3 \%$ of patients). Of these, two had subacute and two acute DVT. Also, two of the four patients exhibited risk factors for DVT.

A total of 37 stents were placed in 21 patients. Thirtyone received nitinol stents; four stainless steel stents and two XL nitinol stents. The mean stent diameter was $12 \pm 2 \mathrm{~mm}$ (range, 10-16 $\mathrm{mm}$ ) and the mean stent length $48.8 \pm 11.6 \mathrm{~mm}$ (range, $40-100 \mathrm{~mm}$ ). Seven patients were implanted with single stent, twelve with two stents, and two with three stents. After each procedure, venography revealed good common iliac vein patency and flow of contrast material (Fig. 1 and Fig. 2).

The clinical and radiological mean follow-up time was $15.9 \pm 16.1$ months (range, 6-48 months). During the postoperative period, 19 of 23 patients $(82.6 \%)$ exhibited complete resolution of symptoms, and symptomatic
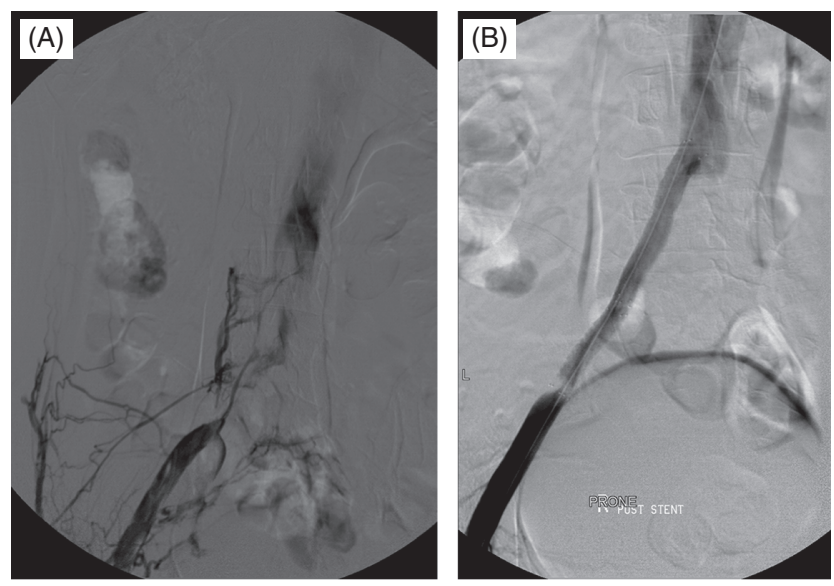

Fig. 2 A 42-year-old female with acute left lower leg deep vein thrombosis. (A) Venography performed after thromboaspiration and catheter-directed thrombolysis revealed residual occlusion of the left common iliac vein. (B) Venography performed after completion of the procedure revealed a patent stent and good flow of contrast material through the stent into the inferior vena cava.

improvement was observed in 3 (13\%). Follow-up performed in the first postoperative month showed that leg swelling decreased in DVT patients, and pain was relieved. Leg edema in those with chronic venous hypertension decreased and varicose veins collapsed. Rectal bleeding in the single patient with this complaint ceased and rectal mucosal congestion seemed to be retarded upon rectoscopic examination. Three patients who could not be completely recanalized to relieve femoral vein thrombosis exhibited moderate levels of symptomatic improvement; these patients had post-thrombotic DVI (deep vein insufficiency). One patient who could not be recanalized 
because of occlusion of the LCIV did not exhibit any symptomatic improvement.

Follow-up CDU and CT venography revealed that patents lumen of the stents $(90.4 \%)$ in 19 of the 21 patients who received stents. Restenosis $(9.6 \%)$ of stents occurred in two patients at 6 and 12 months postoperatively. In one of the patients in which restenosis was developed Factor V Leiden mutation which is known to be a risk factor for DVT was present. In the other patient postoperative irregular anticoagulant medicament usage story was present. One patient was treated via repeat stent implantation and balloon dilatation; the symptoms improved. The other patient received no intervention as no significant clinical finding was apparent. Clinical and radiological signs, treatment details, and follow-up data on all patients are shown in Table 1 . Results at the last follow up visit are shown in Table 2.

No patient experienced PE during follow-up. No procedure-related mortality or major complication was noted. Four patients $(17.4 \%)$ developed minor complications; these were controllable bleeding episodes at the vein access sites.

\section{Discussion}

May-Thurner syndrome, also termed the "syndrome of compression of the iliac vein", is caused by an anatomical anomaly in which the left common iliac vein is compressed by the overlying right common iliac artery. In 1956, May and Thurner found that $22 \%$ of adult subjects studied exhibited vascular thickening of the left iliofemoral venous wall at the point where the right common iliac artery (RCIA) intersected the LCIV. The cited authors concluded that the obstructive lesion, which they termed a "spur", was analogous to a callus in that formation thereof probably required chronic repetitive irritation, identified to be intermittent compression of the LCIV region lying between the pulsating RCIA and the vertebral body. Chronic vibratory pulsation of the vein may damage the intimal wall, in turn causing intimal proliferation and venous thrombosis. ${ }^{1,2)}$ Further work showed that patients with venous spurs could remain asymptomatic for extended periods of time because collateral forms of venous drainage developed. ${ }^{9)}$ Thus, spur formation was considered to be irreversible. Extensive pelvic venous collateral flow circumventing the abnormal LCIV segment is a hallmark of MTS. ${ }^{9}{ }^{10)}$ Several anatomical variants of MTS have been described; these include LCIV compression by the LCIA; right common iliac vein (RCIV) compression by the right internal iliac artery; and right-sided MTS developing where the LCIA compresses the RCIV. ${ }^{11,12)}$ Cadaveric studies showed that the prevalence of LCIV compression ranged from $14-32 \%,{ }^{1,13)}$ but was present in $2-5 \%$ of patients evaluated after complaining of lower limb venous disorders. ${ }^{14)}$ This difference between postmortem and symptomatic compression levels clearly shows that LCIV compression is necessary, but not sufficient, to trigger MTS. ${ }^{15)}$

In the past, MTS was thought to be rare, but the reported frequency of the condition has risen upon development of catheter-directed endovascular treatment of lower-extremity DVT. The likely reason is that the iliac veins are better visualized when venography is performed using vascular sheaths placed in increasingly central veins. ${ }^{6)}$

MTS has been reported to be more frequent in females, as, indeed, we found. No explanation of the female predominance of IVCS has yet been advanced. ${ }^{11,16)}$

MTS most frequently presents as chronic venous insufficiency, and DVT, in young-to- middle-aged females, and is diagnosed in $18-49 \%$ of cases presenting with left lower limb DVT. ${ }^{4,5,6,11)}$ The presence of MTS is suggested by a history of recurrent left lower-extremity DVT and symptoms associated with chronic venous hypertension including varicosities; venous ulcers; lower limb swelling; and pain in the inguinal, calf, or upper leg regions. Also, acute thrombosis may develop in patients at high risk of DVT. DVT attributable to IVCS was reported to occur predominantly in young-to-middle-aged females (aged 20-40 years). Pregnancy and postpartum status are known risk factors for DVT. ${ }^{15)}$ In our present study, 14 patients presented with acute or subacute DVT, and were of older age. One patient was of postpartum status and had a history of eclampsia, and two were of early postoperative status, having undergone surgery for removal of lower-extremity venous varices. Also, patients with IVCS are at increased risk of DVT. ${ }^{15)}$ Significant iliac vein compression may thus be but one risk factor of many favoring DVT development.

Several imaging modalities are useful in MTS diagnosis. CDU is the first-line method used to screen patients with CVI disorders, and can detect DVT. ${ }^{17,18)}$ However, the technique cannot accurately identify venous spurs or compression of the LCIV. If MTS is suspected in patients subjected to ultrasonography, cross-sectional imaging (CT venography, magnetic resonance angiography) or venography should be used to accurately visualize the pelvic region. ${ }^{19,20)}$

Surgical techniques have had mixed success in management of MTS because of high rates of morbidity and variation in patency rates. Surgical options include Palma's crossover technique; relocation of the RCIA behind the LCIV or inferior vena cava; and vein-patch angioplasty with removal of intraluminal obstructions and encasement of the LCIV in polytetrafluoroethylene (ePTFE) grafts. ${ }^{21)}$ Jost, et al. (2001) showed that surgical reconstruction 


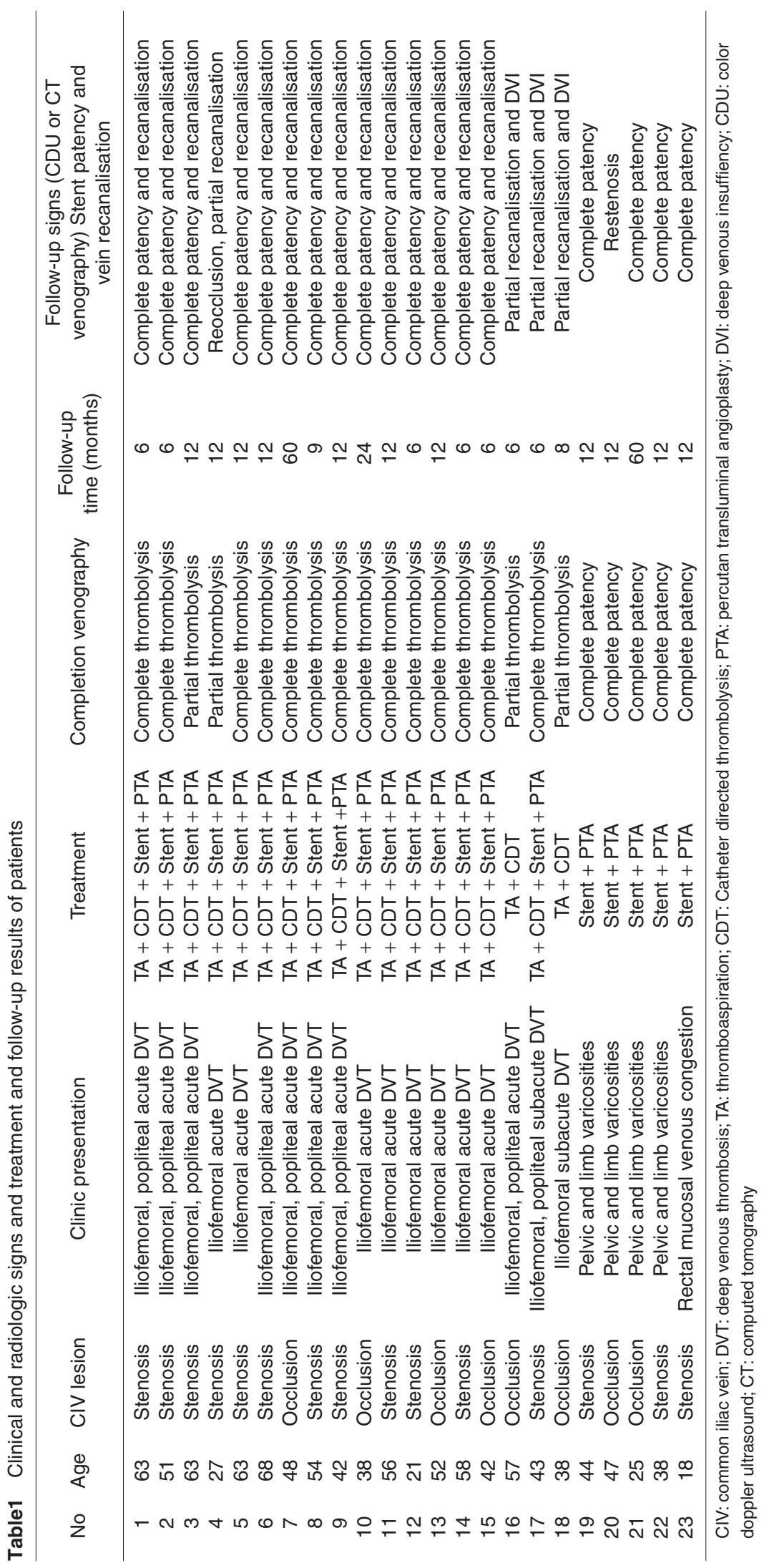


Table 2 Results at the last follow up visit

\begin{tabular}{lcc}
\hline Symtomatic status & Number of Patients (n: 23) & Percentage (\%) \\
Complete resolution & 19 & 82.6 \\
Improvement & 3 & 13 \\
No change & 1 & 4.4 \\
Stent patency & Number of patients (n: 21) & Percentage (\%) \\
Complete & 19 & 90.4 \\
Restenosis & 2 & 9.6 \\
\hline
\end{tabular}

techniques were associated with primary and secondary 3 -year patency rates of $54 \%$ and $62 \%$, respectively. $5,10,21,22)$

The surgical techniques used in another study were both diverse and complicated, including multiple treatments of autologous veins. However, despite such diversity, similar primary and secondary 5 -year patency rates $(42 \%$ and $59 \%$, respectively) were reported in a larger patient group. ${ }^{22)}$

Today, MTS patients rarely undergo highly invasive venous surgical reconstructions because management using endovascular techniques has been relatively successful, and is associated with fewer operative risks. ${ }^{4-8)}$

DVT treatment aims to alleviate acute or chronic symptoms, and to prevent recurrence, PE development, and development of post-thrombotic syndrome. The standard treatment for acute or subacute DVT is anticoagulation therapy, which reduces the rates of recurrence and PE but does not effectively remove clots. ${ }^{23)}$ However, in MTS cases, such management is unlikely to entirely prevent the development of chronic thrombi caused by venous spurs. ${ }^{4,10,24)}$ This is because anticoagulation therapy does not directly treat iliac vein compression or the resulting venous spur; these are the most probable underlying causes of MTS.

Treatment of MTS patients who present with thrombosis has changed dramatically over time; CDT has found increasingly wider applications. Catheter-directed endovascular treatment is more effective in terms of clot removal and symptom improvement, compared to anticoagulation therapy. ${ }^{25)}$ Local infusion of thrombolytic agents at a site of thrombotic occlusion maximizes therapeutic effects while minimizing the risk of major bleeding. Such methods also reduce post-thrombotic morbidity and improve health-related quality-of-life. ${ }^{26)}$

PTA procedures lacking subsequent stent placement have been associated with low patency rates. A 73\% recurrence rate was noted in patients with acute leftsided iliofemoral DVT when the underlying obstruction was not treated via stent placement. ${ }^{4)}$ The chronic venous compression occurring in MTS pateints cannot be relieved via temporary balloon angioplasty. Stents are effective to treat venous obstructions, and are superior to balloon dilatation alone. ${ }^{27)}$ Thus, MTS treatment almost always features the placement of stents exhibiting high radial force. ${ }^{28)}$ Study series featuring iliac vein stenting in MTS patients have increased in number over the past decade and have generally advocated the use of endovascular techniques. ${ }^{5,6,29)}$ Recently, a retrospective review by Titus, et al. identified MTS patients treated via endovenous stenting and showed that the primary patency rates were $88 \%, 78 \%$, and $78 \%$, respectively, at 6,12 , and 24 months postoperatively. ${ }^{30)}$ O'Sullivan, et al. reported that the overall patency rate at 1 year was $79 \% .{ }^{5)}$ Stent placement in iliac veins in combination with thrombus removal affords good short- and mid-term patency especially upon treatment of DVT developing secondarily to MTS. Stent insertion may be important if organic abnormalities are to be treated. ${ }^{10)}$ The possible problems of valve destruction by stents, with subsequent development of venous insufficiency, does not apply to femoral veins because iliac veins lack valves. It has been reported that venous claudication improved after successful stent recanalization in limbs, despite deterioration of the reflux that normalizes venous outflow. Also, calf muscle pump function was enhanced and significant improvements in clinical outcomes were apparent. ${ }^{31)}$

Today, the guidelines of both the Society for Interventional Radiology and the Society of Vascular Surgery recommend iliac vein stenting in the context of external compression. ${ }^{32)}$ We considered that balloon dilatation per se was not adequate for treating our patients. Thus, we initially placed stents in stenotic main iliac vein segments and next used angioplasty balloons to dilate the stents. In tight or narrow segments through which stent carrier shafts could not pass, or if occlusions are present, balloon dilatation can be performed first and stent placement subsequently.

Our results are in accord with those of previously reported series and show that endovascular treatment effectively treats both CVH and DVT caused by MTS; the medium-term patency levels were good.

Patency rates in MTS patients either lacking thrombi or with acute thrombosis were significantly greater than in MTS patients with chronic DVT, showing that early recognition and management of iliac vein compression affords more favorable outcomes. ${ }^{33)}$ It is important to 
diagnose MTS patients in the early stages of disease, thus before DVT appears or becomes chronic. In MTS patients with major DVT risk factors (pregnancy or longterm immobilization), both anticoagulation therapy and refraining from oral comtraceptive use should be suggested.

Some practitioners advocate the use of IVC filters to prevent possible fatal PE events, especially prior to performance of procedures treating CDT or AT. ${ }^{34)}$ Although DVT is common in patients with MTS, such patients rarely present with a PE. Venous spurs appear to afford some protection against PE, particularly when venous stenosis is significant. ${ }^{7)}$ Thus, IVC filter placement is of questionable utility in MTS patients. Vena cava filters can be placed in patients without occlusions who cannot receive anticoagulant treatment. We placed vena cava filters in our early MTS patients who received endovascular treatment, especially prior to thromboaspiration, as has been performed in other patients with acute iliofemoral DVT. However, later, we ceased this practice, as the risk of PE was low. We have not observed symptomatic $\mathrm{PE}$ in any patient before or after treatment.

The limitations of the present study were the retrospective nature of the work, the limited number of MTS patients evaluated, and the loss of some patients to follow-up (limiting our long-term data). Follow-up periods and control numbers were not standardized, and follow-up periods were relatively short. Another potential limitation is the variability in stent type and size. Also, although the use of CDU to assess iliac vein patency is not stressful for patients, the technique has certain limitations. No clinical scale was used for symptomatic evaluation; this was a significant limitation.

As a result, recent reports have shown that endovascular treatment of symptomatic MTS patients with thrombotic or nonthrombotic presentations is both feasible and safe, and improves symptoms. On long-term follow-up, stent patency does not seem to be affected by ongoing extrinsic compression of a vein by the adjacent iliac artery. We obtained excellent immediate results and early patency, with minimal complications, using localized CDT, angioplasty, and stent implantation.

\section{Acknowledgement}

The English in this document has been checked by at least two professional editors, both native speakers of English. For a certificate, please see: http://www.textcheck.com/ certificate/39wsTw

\section{Disclosure Statement}

The authors declare that they have no conflicts of interest.

\section{References}

1) MAY R, THURNER J. The cause of the predominantly sinistral occurrence of thrombosis of the pelvic veins. Angiology 1957; 8: 419-27.

2) Cockett FB, Thomas ML, Negus D. Iliac vein compression.Its relation to iliofemoral thrombosis and the post-thrombotic syndrome. Br Med J 1967; 2: 14-9.

3) Kibbe MR, Ujiki M, Goodwin AL, et al. Iliac vein compression in an asymptomatic patient population. J Vasc Surg 2004; 39: 937-43.

4) Mickley V, Schwagierek R, Rilinger N, et al. Left iliac venous thrombosis caused by venous spur: treatment with thrombectomy and stent implantation. J Vasc Surg 1998; 28: $492-7$.

5) O'Sullivan GJ, Semba CP, Bittner CA, et al. Endovascular management of iliac vein compression (May-Thurner) syndrome. J Vasc Interv Radiol 2000; 11: 823-36.

6) Oguzkurt L, Tercan F, Ozkan U, et al. Iliac vein compression syndrome: outcome of endovascular treatment with longterm follow-up. Eur J Radiol. 2008; 68: 487-92.

7) Hager ES, Yuo T, Tahara R, et al. Outcomes of endovascular intervention for May-Thurner syndrome. Journal of Vascular Surgery: Venous and Lymphatic Disorders, Volume 1, Issue 3, Pages 270-275

8) Heijmen RH, Bollen TL, Duyndam DAC, et al. Endovascular venous stenting in May-Thurner syndrome. J Cardiovasc Surg 2001; 42: 83-7.

9) Cockett FB, Thomas ML, Negus D. Iliac vein compression.Its relation to iliofemoral thrombosis and the post-thrombotic syndrome. Br Med J 1967; 2: 14-9.

10) Patel NH, Stookey KR, Ketcham DB, et al. Endovascular management of acute extensive iliofemoral deep venous thrombosis caused by May-Thurner syndrome. J Vasc Interv Radiol 2000; 11: 1297-302.

11) Hassell DR, Reifsteck JE, Harshfield DL, et al. Unilateral left leg edema: a variation of the May-Thurner syndrome. Cardiovasc Intervent Radiol 1987; 10: 89-91.

12) Abboud G, Midulla M, Lions C, et al. "Right-sided" May-Thurner syndrome. Cardiovasc Intervent Radiol. 2010; 33: 1056-9.

13) Ehrich WE, Krumbhaar EB. A frequent obstructive anomaly of the mouth of the left common iliac vein. American Heart Journal 1943; 26: 18-31.

14) Posner C, Owen M, Melhem N, et al. An acutely swollen leg. Clin Med 2010; 10: 484-6.

15) National Institute of Health. Prevention of venous thrombosis and pulmonary embolism. NIH Consensus Development. JAMA1986; 256: 744-9.

16) Brazeau NF, Harvey HB, Pinto EG, et al. MayThurner syndrome: diagnosis and management. Vasa. 2013; 42: 96-105.

17) Ahmed HK, Hagspiel KD. Intravascular ultrasonographic findings in May-Thurner syndrome (iliac vein compression syndrome). J Ultrasound Med 2001; 20: 251-6.

18) Chung JW, Yoon CJ, Jung SI, et al. Acute iliofemoral deep vein thrombosis: evaluation of underlying anatomic abnormalities by spiral CT venography. J Vasc Interv Radiol 2004; 15: 249-56. 
19) Oguzkurt L, Tercan F, Pourbagher MA, et al. Computed tomography findings in 10 cases of iliac vein compression (May-Thurner) syndrome. Eur J Radiol. 2005; 55: 421-5.

20) Gurel K, Gurel S, Karavas E, et al. Direct contrastenhanced MR venography in the diagnosis of May-Thurner syndrome. Eur J Radiol 2011; 80: 533-6.

21) Jost CJ, Gloviczki P, Cherry KJ Jr, et al. Surgical reconstruction of iliofemoral veins and the inferior vena cava for nonmalignant occlusive disease. J Vasc Surg 2001; 33: 320-7; discussion 327-8.

22) Garg N, Gloviczki P, Karimi KM, et al. Factors affecting outcome of open and hybrid reconstructions for nonmalignant obstruction of iliofemoral veins and inferior vena cava. J Vasc Surg 2011; 53: 383-93.

23) AbuRahma AF, Perkins SE, Wulu JT, et al. Iliofemoral deep vein thrombosis: conventional therapy versus lysis and percutaneous transluminal angioplasty and stenting. Ann Surg 2001; 233: 752-60.

24) Nazir SA, Ganeshan A, Nazir S, et al. Endovascular treatment options in the management of lower limb deep venous thrombosis. Cardiovasc Intervent Radiol 2009; 32: 861-76.

25) Mewissen MW, Seabrook GR, Meissner MH, et al. Catheter-directed thrombolysis for lower extremity deep venous thrombosis: report of a national multicenter registry. Radiology 1999; 211: 39-49.

26) Comerota AJ, Throm RC, Mathias SD, et al. Catheterdirected thrombolysis for iliofemoral deep venous thrombosis improves health-related quality of life. J Vasc Surg 2000; 32: 130-7.
27) Kim JY, Choi D, Guk Ko Y, et al. Percutaneous treatment of deep vein thrombosis in May-Thurner syndrome. Cardiovasc Intervent Radiol 2006; 29: 571-5.

28) Neglén P, Hollis KC, Olivier J, et al. Stenting of the venous outflow in chronic venous disease: long-term stent-related outcome, clinical, and hemodynamic result. J Vasc Surg 2007; 46: 979-90.

29) Binkert CA, Schoch E, Stuckmann G, et al. Treatment of pelvic venous spur (May-Thurner syndrome) with selfexpanding metallic endoprostheses. Cardiovasc Intervent Radiol 1998; 21: 22-6.

30) Titus JM, Moise MA, Bena J, et al. Iliofemoral stenting for venous occlusive disease. J Vasc Surg 2011; 53: 706-12.

31) Delis KT, Bountouroglou D, Mansfield AO. Venous claudication in iliofemoral thrombosis: long-term effects on venous hemodynamics, clinical status, and quality of life. Ann Surg 2004; 239: 118-26.

32) Meissner MH, Gloviczki P, Comerota AJ, et al. Early thrombus removal strategies for acute deep venous thrombosis: clinical practice guidelines of the Society for Vascular Surgery and the American Venous Forum. J Vasc Surg 2012; 55: 1449-62.

33) Lou WS, Gu JP, He X, et al. Endovascular treatment for iliac vein compression syndrome: a comparison between the presence and absence of secondary thrombosis. Korean J Radiol. 2009; 10: 135-43.

34) Kinney TB. Update on inferior vena cava filters. J Vasc Interv Radiol 2003; 14: 425-40. 